\title{
Beta-blockers and COPD: the show must go on
}

\author{
Jose Luis Lopez-Campos ${ }^{1,2}$, Eduardo Márquez-Martín ${ }^{1}$ and Ciro Casanova ${ }^{3}$
}

Affiliations: ${ }^{1}$ Unidad Médico-Quirúrgica de Enfermedades Respiratorias, Instituto de Biomedicina de Sevilla (IBiS), Hospital Universitario Virgen del Rocío/Universidad de Sevilla, Seville, Spain. ${ }^{2}$ CIBER de Enfermedades Respiratorias (CIBERES), Instituto de Salud Carlos III, Madrid, Spain. ${ }^{3}$ Pulmonary Dept, Hospital Universitario Nuestra Señora de Candelaria, Santa Cruz de Tenerife, Spain.

Correspondence: Jose Luis Lopez-Campos, Hospital Universitario Virgen del Rocio, Avda. Manuel Siurot, s/n. 41013 Seville, Spain. E-mail: Icamposdsepar.es

0 @ERSpublications

Current evidence on beta-blockers efficacy and safety in COPD is encouraging but limited with future studies needed http://ow.ly/IaNL301YANI

Identification of comorbidities is now recognised as one of the pillars for a comprehensive clinical evaluation in chronic obstructive pulmonary disease (COPD) [1]. Specifically, the burden of coexisting cardiovascular disease in COPD has gained significant attention, with specific algorithms being developed for its clinical detection [2]. The relationship between the heart and COPD is of clinical relevance not only for the well-documented relationship between the two organs [3], but also for the potential mutual influence of treatments. The interactions between oral beta-blockers and inhaled $\beta$-adrenergic drugs pose significant challenges for clinicians involved in the management of patients with chronic cardiorespiratory conditions. In particular, the use of beta-blockers in COPD remains the subject of ongoing controversy [4].

In this issue of the European Respiratory Journal, LIPWORTH et al. [5] provide a comprehensive summary on the use of beta-blockers in patients with COPD. In their narrative review, the authors disentangled the complex links between COPD and the heart, reviewed the available data on the use of beta-blockers for reducing exacerbations and mortality, and summarised the unmet needs in the field, with special reference to a more in-depth knowledge of how different beta-blockers can affect pulmonary function based on their pharmacology. Despite published reports of physicians being reluctant to prescribe beta-blockers in COPD patients with a history of myocardial infarction or coexisting heart failure, the authors conclude that there are compelling reasons for the use of cardioselective beta-blockers in this patient group.

In order to understand the potential implications of beta-blockers on COPD a brief review of the lung innervation and anatomical distribution of receptors in the lung is required. In humans, the direct sympathetic innervation of the airway smooth muscle is very poor [6]. Somewhat counterintuitively, however, the density of $\beta$-adrenergic receptors is markedly high and their expression can be identified in different cell types. In the human lung, $\beta$-adrenergic receptors on smooth muscle, epithelial and mast cells are of the $\beta_{2}$-subtype, whose density increases towards the peripheral airways. By contrast, human submucosal glands and alveolar wall cells also express $\beta_{1}$-receptors $[7,8]$. In the healthy heart, $\beta_{1^{-}}$and $\beta_{2}$-adrenergic receptors coexist in a 4:1 ratio. However, in the failing heart, $\beta_{1}$-adrenergic receptor numbers decrease, paralleled by an increase in $\beta_{2}$-adrenergic receptors, ultimately resulting in a 3:2 ratio [9]. In this scenario, the occurrence of interactions may not be negligible. In general, the effects of drugs that act on adrenergic receptors depend both on the intrinsic pharmacological properties of the drug (including the $\beta_{1} / \beta_{2}$ ratio) and its bioavailability (intended to be high in systemic beta-blockers but low in inhaled $\beta$-agonists with an adequate inhalation technique).

Starting from these premises, multiple evidence excellently summarised by LipwORTH et al. [5] merits comment. First, a point that is worth considering is the potential effect of beta-blockers in the prevention

Received: June 202016 | Accepted: June 212016

Conflict of interest: Disclosures can be found alongside this article at erj.ersjournals.com

Copyright OERS 2016 
of exacerbations in stable COPD patients. The main difficulty in the interpretation of the available data is that the published reports are mainly retrospective analyses of clinical records. It is well-known that these studies are not only limited by potential biases and confounders that were not taken into account but also influenced by the analytical approach used. Additionally, the identification of COPD cases and COPD exacerbations in clinical records has been questioned [10]. The discrimination between a COPD exacerbation and a decompensation of coexisting cardiac or respiratory diseases may be problematic in daily clinical practice. The problem is obviously even more difficult when clinical datasets are retrospectively reviewed. It should also be kept in mind that a worsening of respiratory symptoms in a COPD patient does not invariably reflect a COPD exacerbation [11]. Accordingly, use of the term complications rather than exacerbations has been proposed [12]. Strict diagnostic algorithms have been proposed for the adequate identification and analysis of stable [13] and exacerbated COPD patients in clinical records from primary [14] and secondary care [15].

Interestingly, one prospective study conducted in the COPDGene cohort assessed whether the use of beta-blockers can decrease the occurrence of exacerbations [16]. The results of this longitudinal study indicated that the use of beta-blockers was associated with a significant reduction in COPD exacerbations, independent of the severity of airflow obstruction. A cross-sectional study of COPD patients with chronic heart failure or coronary artery disease reported similar findings [17]. It should be noted, however, that another study found opposite results in patients in Global Initiative for Chronic Obstructive Lung Disease stages 3 and 4 on home oxygen therapy [18]. To make things more complex, different small studies have shown a decrease in different lung function parameters with the use of the cardioselective beta-blocker bisoprolol $[19,20]$. Therefore, the question as to whether beta-blockers may decrease lung function while reducing the frequency of exacerbations remains open and researchers should consider whether we are seeing an effect on COPD exacerbations or on cardiac events. Taken together, there is retrospective and some longitudinal evidence that beta-blockers can decrease acute adverse clinical events in COPD patients. Further research efforts are needed to dissect the exact impact of beta-blockers on COPD exacerbations, respiratory symptoms and lung function parameters. The $\beta$ LOCK COPD ( $\beta$-blockers for the prevention of acute exacerbations of chronic obstructive pulmonary disease) trial is currently being conducted and its results will surely shed more light on this issue [21].

Second, another source of controversy is the potential prognostic significance of beta-blocker use in patients with COPD. Beside the abovementioned issue related to exacerbations, the effect of beta-blockers on cardiac function should be considered separately in patients with and without COPD. A number of studies have investigated the impact of COPD on cardiac function. Patients with COPD but no overt cardiovascular disease show signs of left ventricle concentric remodelling [3]. Moreover, COPD has emerged as a significant risk factor for ventricular arrhythmias and sudden cardiac death [22]. Nonetheless, it remains unclear whether the cardiac effect of beta-blockers may differ in patients with COPD compared with those without. Consequently, it is still debatable whether beta-blockers reduce the risk in COPD only through their cardiovascular effects or due to a potential respiratory effect and, if this is so, by which mechanism. Interestingly, the prospective COPDGene study failed to identify a statistically significant association between the use of beta-blockers and mortality in COPD [16]. This relationship needs to be prospectively evaluated in longitudinal trials with rigorously established clinical end-points.

Third, the potential usefulness of beta-blockers during COPD exacerbations merits consideration. Studies based on the analysis of clinical datasets suggest that the use of beta-blockers in patients admitted for a COPD exacerbation is significantly associated with a reduced mortality [23]. Beside the abovementioned issue related to the retrospective identification of exacerbations, the diagnosis of an exacerbation represents another challenge. The European COPD Audit revealed that $40.6 \%$ of patients admitted to hospital and subsequently discharged with a diagnosis of a COPD exacerbation did not have a previous diagnosis of COPD according to current guidelines. In addition, $12.9 \%$ of these subjects had previously undergone spirometry that did not reveal obstructive findings [24]. In real-world practice, several patients with COPD require hospital admission because of cardiac disorders. The cardiovascular risk of COPD patients is also markedly higher during exacerbations, which are accompanied by increased levels of biomarkers of cardiac injury [25] and carry negative prognostic implications [26, 27]. Beta-blockers may limit these phenomena and improve survival in patients admitted for exacerbations [23]. Again, further research is needed to investigate whether the positive impact of beta-blockers should be attributed to their effect on cardiac function and/or respiratory exacerbations per se.

Another point examined by LIPWORTH et al. [5] is the potential crossover efficacy between systemic beta-blockers and inhaled medications. Pharmacological treatment of COPD exerts beneficial effects on both cardiac function and lung vasculature, which may in turn contribute to the favourable effects of inhaled therapies [28]. Even at low doses, the $\beta_{1}$-selective antagonist atenolol may protect against the chronotropic, inotropic and electrocardiographic effects of inhaled $\beta$-agonists [29]. Furthermore, the 
administration of beta-blockers in a murine model of asthma resulted in an increased pulmonary $\beta$-receptor density and moderate anti-inflammatory effects [30,31], suggesting that this drug class may increase the response to inhaled asthma medications. Another interesting observation is that long-acting muscarinic antagonists can reduce the risk of bronchoconstriction even when nonselective beta-blockers are used in patients with asthma [32]. If similar results are reported for patients with COPD, future trials specifically focusing on this new efficacy end-point will be needed.

Finally, LiPWORTH et al. [5] correctly point out that the presence of untreated or undetected cardiovascular disease may influence both the clinical presentation and outcomes of COPD. Patients with silent cardiovascular disease may specifically represent a significant clinical challenge because they may be under-recognised. In addition, the acquisition of echocardiography images may be problematic owing to lung hyperinflation. The development of a standardised diagnostic work-up for the detection of cardiovascular disease in patients with COPD may be helpful to reduce the risk of underdiagnosis.

In summary, most of the available evidence indicates that beta-blockers are generally safe in COPD patients. Nonetheless, additional longitudinal studies are needed before more definitive recommendations can be made. Continuing research into the complex relationships of beta-blockers in COPD will be obviously required. All we can say, in remembrance of Queen's international hit from the "Innuendo" album (released in October 1991), is that "the show must go on" in the field. Future points that specifically need to be clarified include: 1) standardisation of the diagnostic approach for the detection of cardiac dysfunction in COPD; 2) the safety of beta-blockers in COPD, with a special focus on the differences between distinct molecules; and 3) the effect of beta-blockers in terms of exacerbations, symptoms, lung function parameters and prognosis. With these data to hand, the prescription of beta-blockers to COPD patients will take place with a reasonable degree of confidence.

\section{References}

1 Marin JM, Cote C, Casanova C, et al. Simplifying the guidelines: the 10 COPD commandments. Arch Bronconeumol 2016; 52: 179-180.

2 Cazzola M, Calzetta L, Matera MG, et al. Chronic obstructive pulmonary disease and coronary disease: COPDCoRi, a simple and effective algorithm for predicting the risk of coronary artery disease in COPD patients. Respir Med 2015; 109: 1019-1025.

3 Pelà G, Li Calzi M, Pinelli S, et al. Left ventricular structure and remodeling in patients with COPD. Int J Chron Obstruct Pulmon Dis 2016; 11: 1015-1022.

4 Malerba M, Montuschi P, Radaeli A, et al. Role of beta-blockers in patients with COPD: current perspective. Drug Discov Today 2015; 20: 129-135.

5 Lipworth B, Wedzicha J, Devereux G, et al. Beta-blockers in COPD: time for reappraisal. Eur Respir J 2016; 48: 880-888.

6 Belvisi MG. Overview of the innervation of the lung. Curr Opin Pharmacol 2002; 2: 211-215.

7 Ikeda T, Anisuzzaman AS, Yoshiki H, et al. Regional quantification of muscarinic acetylcholine receptors and beta-adrenoceptors in human airways. Br J Pharmacol 2012; 166: 1804-1814.

8 van der Velden VH, Hulsmann AR. Autonomic innervation of human airways: structure, function, and pathophysiology in asthma. Neuroimmunomodulation 1999; 6: 145-159.

9 Najafi A, Sequeira V, Kuster DW, et al. Beta-adrenergic receptor signalling and its functional consequences in the diseased heart. Eur J Clin Invest 2016; 46: 362-374.

10 Stein BD, Bautista A, Schumock GT, et al. The validity of International Classification of Diseases, Ninth Revision, Clinical Modification diagnosis codes for identifying patients hospitalized for COPD exacerbations. Chest 2012; 141: 87-93.

11 Beghé $\mathrm{B}$, Verduri A, Roca $\mathrm{M}$, et al. Exacerbation of respiratory symptoms in COPD patients may not be exacerbations of COPD. Eur Respir J 2013; 41: 993-995.

12 Lopez-Campos JL, Calero C, Lopez-Ramirez C. Exacerbations or complications? Redefining the concepts in COPD. Int J Clin Pract 2014; 68: 1048.

13 Smidth M, Sokolowski I, Kærsvang L, et al. Developing an algorithm to identify people with chronic obstructive pulmonary disease (COPD) using administrative data. BMC Med Inform Decis Mak 2012; 12: 38.

14 Kerkhof M, Freeman D, Jones R, et al. Predicting frequent COPD exacerbations using primary care data. Int $J$ Chron Obstruct Pulmon Dis 2015; 10: 2439-2450.

15 Rothnie KJ, Müllerová H, Hurst JR, et al. Validation of the recording of acute exacerbations of COPD in UK primary care electronic healthcare records. PLoS One 2016; 11: e0151357.

16 Bhatt SP, Wells JM, Kinney GL, et al. $\beta$-Blockers are associated with a reduction in COPD exacerbations. Thorax 2016; 71: 8-14.

17 Puente-Maestu L, Calle M, Ortega-González A, et al. Multicentric study on the beta-blocker use and relation with exacerbations in COPD. Respir Med 2014; 108: 737-744.

18 Ekström MP, Hermansson AB, Ström KE. Effects of cardiovascular drugs on mortality in severe chronic obstructive pulmonary disease. Am J Respir Crit Care Med 2013; 187: 715-720.

19 Hawkins NM, MacDonald MR, Petrie MC, et al. Bisoprolol in patients with heart failure and moderate to severe chronic obstructive pulmonary disease: a randomized controlled trial. Eur J Heart Fail 2009; 11: 684-690.

20 Mainguy V, Girard D, Maltais F, et al. Effect of bisoprolol on respiratory function and exercise capacity in chronic obstructive pulmonary disease. Am J Cardiol 2012; 110: 258-263.

21 Bhatt SP, Connett JE, Voelker H, et al. $\beta$-Blockers for the prevention of acute exacerbations of chronic obstructive pulmonary disease ( $\beta$ LOCK COPD): a randomised controlled study protocol. BMJ Open 2016; 6: e012292. 
22 Liu Y, Narayanan K, Zhang Z, et al. Chronic obstructive pulmonary disease and risk of sudden cardiac death. Int J Cardiol 2016; 214: 406-407.

23 Dransfield MT, Rowe SM, Johnson JE, et al. Use of beta blockers and the risk of death in hospitalised patients with acute exacerbations of COPD. Thorax 2008; 63: 301-305.

24 Roberts CM, Lopez-Campos JL, Pozo-Rodriguez F, et al. European hospital adherence to GOLD recommendations for chronic obstructive pulmonary disease (COPD) exacerbation admissions. Thorax 2013; 68: 1169-1171.

25 Chang CL, Robinson SC, Mills GD, et al. Biochemical markers of cardiac dysfunction predict mortality in acute exacerbations of COPD. Thorax 2011; 66: 764-768.

26 Høiseth AD, Omland T, Karlsson BD, et al. Standardized evaluation of lung congestion during COPD exacerbation better identifies patients at risk of dying. Int J Chron Obstruct Pulmon Dis 2013; 8: 621-629.

27 Pavasini R, d'Ascenzo F, Campo G, et al. Cardiac troponin elevation predicts all-cause mortality in patients with acute exacerbation of chronic obstructive pulmonary disease: systematic review and meta-analysis. Int J Cardiol 2015; 191: 187-193.

28 Stone IS, Barnes NC, James WY, et al. Lung deflation and cardiovascular structure and function in chronic obstructive pulmonary disease. A randomized controlled trial. Am J Respir Crit Care Med 2016; 193: 717-726.

29 Newnham DM, Wheeldon NM, Lipworth BJ, et al. Single dosing comparison of the relative cardiac beta 1/beta 2 activity of inhaled fenoterol and salbutamol in normal subjects. Thorax 1993; 48: 656-658.

30 Callaerts-Vegh Z, Evans KL, Dudekula N, et al. Effects of acute and chronic administration of beta-adrenoceptor ligands on airway function in a murine model of asthma. Proc Natl Acad Sci USA 2004; 101: 4948-4953.

31 Nguyen LP, Omoluabi O, Parra S, et al. Chronic exposure to beta-blockers attenuates inflammation and mucin content in a murine asthma model. Am J Respir Cell Mol Biol 2008; 38: 256-262.

32 Short PM, Anderson WJ, Williamson PA, et al. Effects of intravenous and oral $\beta$-blockade in persistent asthmatics controlled on inhaled corticosteroids. Heart 2014; 100: 219-223. 